\section{VENTICINQUE NOVEMBRE AL NIDO un progetto di sensibilizzazione contro la violenza di genere fisica e simbolica su donne e bambine/i}

Riassunto: Perché parlare di violenza di genere in un nido d'infanzia? I genitori delle bambine/i che frequentano il nido sono interlocutori importanti, con cui confrontarsi sui temi educativi. II progetto si rivolge a donne e vomini che a vario titolo frequentano il nido per sensibilizzarli su un fenomeno che non va relegato al silenzio. Occorre dare voce a chi non ne ha per aiutare a vedere possibilità di evoluzione. E' importante dare alle famiglie di bambini/e piccoli/e, occasioni per riflettere su questi temi. Perché e' fin dalla prima infanzia che si trasmettono e si radicano stereotipi di genere ed e' al nido e nelle scuole che occorre far crescere una cultura di valorizzazione delle differenze. A partire da queste convinzioni i nidi d'infanzia San Donato e Viganò del Comune di Bologna, in occasione del 25 novembre, giornata internazionale contro la violenza sulle donne, promuovono da anni iniziative di sensibilizzazione. Ogni 25 novembre le famiglie sono invitate a trascorrere la giornata al nido insieme alle bambine/i. Durante l'accoglienza viene distribuito materiale informativo della "Casa delle donne per non subire violenza" ed allestito uno spazio con poesie sul tema. Nella mattinata viene proposta una performance, creata dalle educatrici, a cui segue un momento laboratoriale che coinvolge bambini/e e genitori. La performance, carica di elementi simbolici, offre suggestioni legate al tema della giornata, caratterizzata da una forte condivisione di pensieri ed emozioni.

Parole chiave: Feminismo. Contra la Violenza. Nido. 25 november.

\section{Vinte e cinco de novembro na creche: um projeto de sensibilização contra a violência de gênero física e simbólica contra as mulheres e as crianças}

Resumo: Por que falar sobre a violência de gênero em uma creche? Os pais e as mães de crianças que frequentam a creche são interlocutores importantes, com os quais confrontamos a tematica da educação. O projeto destina-se a homens e mulheres que por vários motivos frequentam a creche e busca sensibilizá-los para um fenômeno que não deve ser relegado ao silêncio. Temos de dar voz a quem não a tem e ajudálo/a a ver as possibilidades de mudança. É importante portanto, dar às famílias de crianças pequenas a oportunidades para refletir sobre estas questões. Por que é desde a primeira infância, que são transmitidos e estão enraizados estereótipos de gênero e é na creche e nas pré-escolas que precisa crescer uma cultura de valorização das diferenças. A partir destas convicções, nas creches San Donato e a creche Viganò no município de Bolonha, por ocasião do 25 de Novembro, Dia Internacional Contra a Violência Contra as Mulheres, promovemos há anos eventos de sensibilização. Todo dia 25 de novembro as famílias são convidadas a passar o dia na creche junto com as crianças. Durante o acolhimento é distribuído material informativo da "Casa delle donne per non subire violenza" (Casa das Mulheres para não sofrerem violência) e é criado um espaço com poemas sobre o tema. Na parte da manhã, propomos uma

1 Graduada em Pedagogia. Educadora de creche da prefeitura de Bologna.

${ }^{2}$ Graduada em Pedagogia. Educadora de creche da prefeitura de Bologna. E-mail: mauriziaquerciagrossa@alice.it 
performance artistica, elaborada pelas professoras com uma oficina envolvendo as crianças, as mães e os pais. A performance, repleta de elementos simbólicos, proporciona alternativas relacionadas ao tema do dia, marcadas por uma forte partilha de pensamentos e emoções.

Palavras chaves: Feminismo. Contra violência. 25 de novembro. Creche.

\section{November Twenty-fifth in the daycare center. A sensitive project against physical and symbolic gender violence on women and children}

Abstract: Why talk about gender violence in an environment like the day care center? Parents of children who attend the day care center are very important subjects with which to share educational concepts. This project is dedicated to women and men who attend the daycare center. It has the purpose to discuss this phenomenon that must not be confined in silence. It is necessary to give a voice to those who have none, for helping everyone and, finally, see emotional changes. It is very important, to give occasions to baby's families for thinking about these themes. The first step should be done from the babyhood, just in the moment when gender stereotypes are built and find deep roots. This is the reason, why in the daycare center and then in the preschools, it is necessary to improve a culture based on the appreciation of differences. Starting from this belief, to mark the 25th of November, International Day against violence on women, San Donato and Viganò daycare centers, located in Bologna, promote, for years, awareness-raising initiatives. Every single 25th of November all the families are invited to spend the whole day at the daycare center with the children. During the welcome's moment the organizers hand out information material on "Casa delle donne per non subire violenza" and they set up a space with themed poems. During that morning there is a performance, created by the educators, followed from a workshop moment which involves both children than parents. Every performance chosen is full of symbols and gives sensations related to content of the day, a day based on the strong sharing of thoughts and emotions.

Keywords: Feminism. Day Care Center. Against Violence. November $25^{\text {th }}$.

Perché parlare di violenza di genere in un nido d'infanzia? I genitori delle bambine/i che frequentano il nido sono interlocutori importanti, con cui confrontarsi sui temi educativi. Il progetto si rivolge a donne e uomini che a vario titolo frequentano il nido per sensibilizzarli su un fenomeno che non va relegato al silenzio. Occorre dare voce a chi non ne ha, dare visibilità, stimolare reazioni, aiutare a vedere possibilità di evoluzione. E' importante dare ai genitori di bambini/e piccoli/e, occasioni per riflettere su questi temi e promuovere un cambiamento culturale, per contrastare discriminazioni e pregiudizi legati a stereotipi di genere, perché e’ fin dalla prima infanzia che si trasmettono e si radicano, ed e' al nido e nelle scuole che occorre far crescere una cultura di valorizzazione delle differenze.

“...ci vorrebbe una rieducazione sentimentale. Una pedagogia rivoluzionaria fin dai primi mesi di vita, all'asilo, a scuola, in famiglia. Una nuova educazione che sia capace di modificare l'assegnazione arcaica dei luoghi nelle coscienze" Lydia Cacho 
partire da queste convinzioni i nidi d'infanzia San Donato e Renata.Viganò del Comune di
Bologna, in occasione del 25 novembre, giornata internazionale contro la violenza sulle donne,
promuovono da anni iniziative di sensibilizzazione.

Ogni 25 novembre donne e uomini che frequentano il servizio sono invitate/i a trascorrere la giornata al nido insieme alle bambine/i. Il programma si articola in modi e momenti differenti per dare modo a tutte/i di partecipare direttamente o indirettamente. Durante l'accoglienza viene distribuito materiale informativo della "Casa delle Donne per non subire violenza ONLUS" di Bologna e il programma del "Festival la violenza illustrata" organizzato ogni anno dalla Casa delle Donne di Bologna dal novembre 2006, per promuovere iniziative, dibattiti, cinema, mostre in occasione del 25 novembre (l'iniziativa del nido San Donato è inserita nel programma dal 2012).

Viene inoltre allestito uno spazio con poesie sul tema della violenza di genere raccolte e/o scritte da genitori e/o educatrici. Poesie per testimoniare, per dare voce, per leggere parole nascoste tra silenzi. In giardino diffusione e ascolto di brani musicali inerenti il tema e colazione con karkadè e biscotti. Simbolo costante dell'iniziativa è il colore rosso, rosso come il sangue delle donne e bambini/e vittime di violenza, simbolo di passione, di cuore,di forza, di energia, di chi non abbassa la testa e grida forte il proprio dissenso, di chi re-siste.

Ai genitori viene chiesto di vestire i/le bambini/e con abbigliamento e scarpe rosse, di partecipare indossando vestiti e/o scarpe di colore rosso e di collaborare nella ricerca di oggetti o materiali legati all'iniziativa. Nella mattinata vengono proposte un'installazione e/o una performance,e/o una camminata pensate e create dalle educatrici, a cui a volte segue un momento laboratoriale che coinvolge bambini/e e genitori. Ideazioni per cercare di rappresentare la violenza in modo estetico ed emotivo, per portare a guardare e a riflettere. Per ripensare luoghi del quotidiano in un'ottica di genere, per costruire un immaginario privo di stereotipi e per aprire lo sguardo affrontando senza paura, ma con responsabilità, sensibilità e cultura l'argomento della violenza di genere.

Ogni anno il gruppo di lavoro del nido si interroga sul tema e sulla proposta aderendo alla giornata con tempi e modi differenti nel rispetto delle diverse competenze e sensibilità, inserendo l'iniziativa all'interno del progetto educativo di nido. A partire da questi presupposti i due servizi sopracitati hanno presentato nel corso degli anni proposte differenti.

\section{NIDO D'INFANZIA RENATA VIGANO'}

\section{ADESIONE ALLA GIORNATA INTERNAZIONALE CONTRO LA VIOLENZA SULLE DONNE}

\section{NOVEMBRE 2014 "Scarpe rosse"}

Partendo dalle suggestioni e dalle riflessioni dell'installazione " Zapatos rojas" di Elina Chauvet si è pensato di scegliere le scarpe rosse come simbolo per rappresentare le donne uccise, violate e sparite che 
hanno lasciato il vuoto, ma anche per aprire il cammino per condividere il tema della violenza di genere e contrastare il fenomeno in tutte le sue forme. Al momento dell'accoglienza le scarpe rosse di differenti misure portate dai genitori vengono appese su corde tese da una parte all'altra del corridoio del nido creando visivamente un corteo capace di intrecciare assenza-presenza.

\section{NOVEMBRE 2015}

Simbolo della giornata le scarpe rosse,come segno delle donne vittime della violenza di genere. Un messaggio partito dal nido per dire a tutte le donne di non sentirsi sole di fronte ad atti di violenza. Un paio di scarpe rosse, portate da una donna, un genitore è stato posizionato all'interno di un cerchio di plastica rosso al centro del corridoio del nido. L'iniziativa è stata presentata ai genitori durante l'assemblea di nido, in quell'occasione si è chiesto loro di partecipare portando da casa oggetti rossi di uso domestico (imbuti, asciugamani, bicchieri, piatti...). Gli oggetti come tanti fili rossi che lasciano la casa per arrivare al nido, luogo pubblico ed istituzionale, ci sembravano simboleggiare l'idea di rete sicura che insieme si può costruire per rompere il silenzio. Gli oggetti disposti a spirale sul pavimento sono stati esplorati dai/le bambini/e dei gruppi medi e grandi nella seconda parte della mattinata.

\section{NOVEMBRE 2016}

In continuità con gli anni precedenti le scarpe rosse sono simbolo dell'iniziativa. Un'altra donna, un genitore ha portato un paio di scarpe rosse che è stato posizionato all'interno di un cerchio di plastica al centro del corridoio del nido, I genitori hanno partecipato all'iniziativa portando poesie scritte da donne sul tema della violenza che al nido sono state stampate su fogli rossi e posizionate sul muro lungo il corridoio, e/o tracce grafiche create a casa insieme ai/le loro bambini/e .All'interno della sezione piccoli è stata allestita una vasca contenente palline di plastica rosse dove i/le bambini/e vestiti di rosso hanno nuotato rilasciando emozioni tra sguardi e gesti.

\section{NOVEMBRE 2017}

Il nido nella giornata del 24 è rimasto chiuso per problemi tecnici legati alla struttura, ospitato all'interno di un altro servizio è riuscito a condividere un momento di sensibilizzazione sulla violenza di genere. Simbolo dell'iniziativa il colore rosso 
Figura 1. Piccola mostra fotografica: Venticinque Novembre al Nido

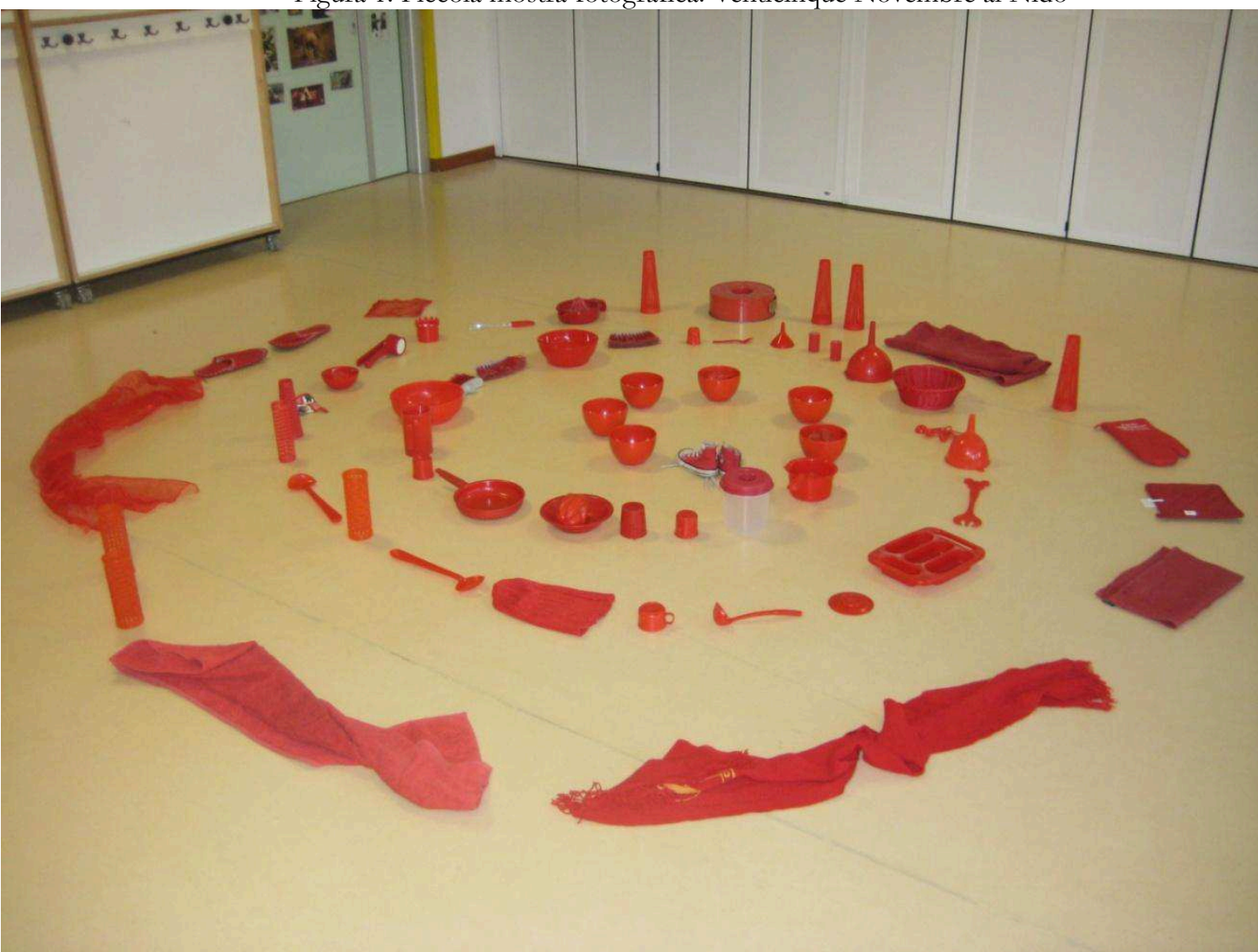

Fonte: Le autrice

Figura 2. Piccola mostra fotografica: Venticinque Novembre al Nido

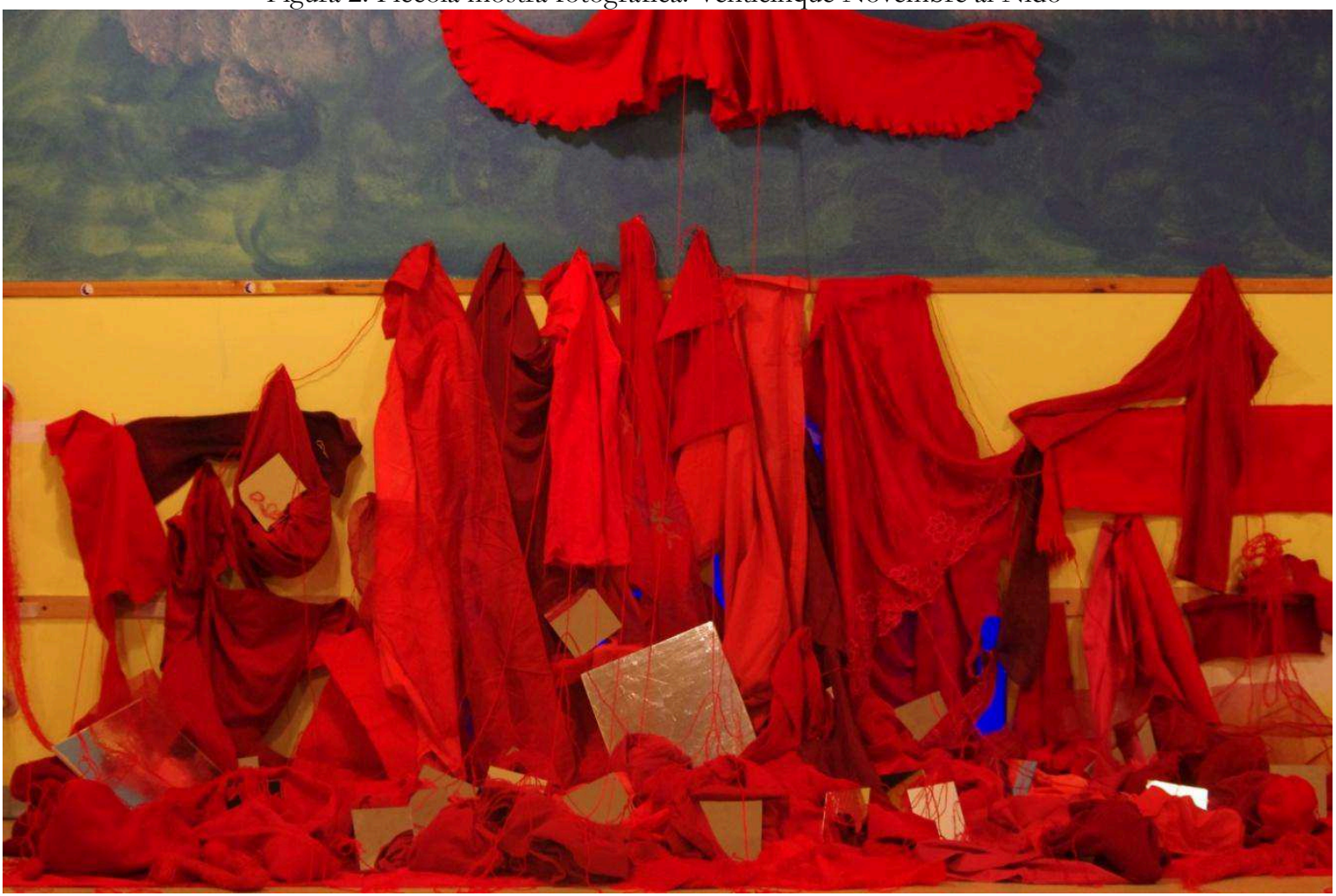

Fonte: Le autrice 
Figura 3. Piccola mostra fotografica: Venticinque Novembre al Nido

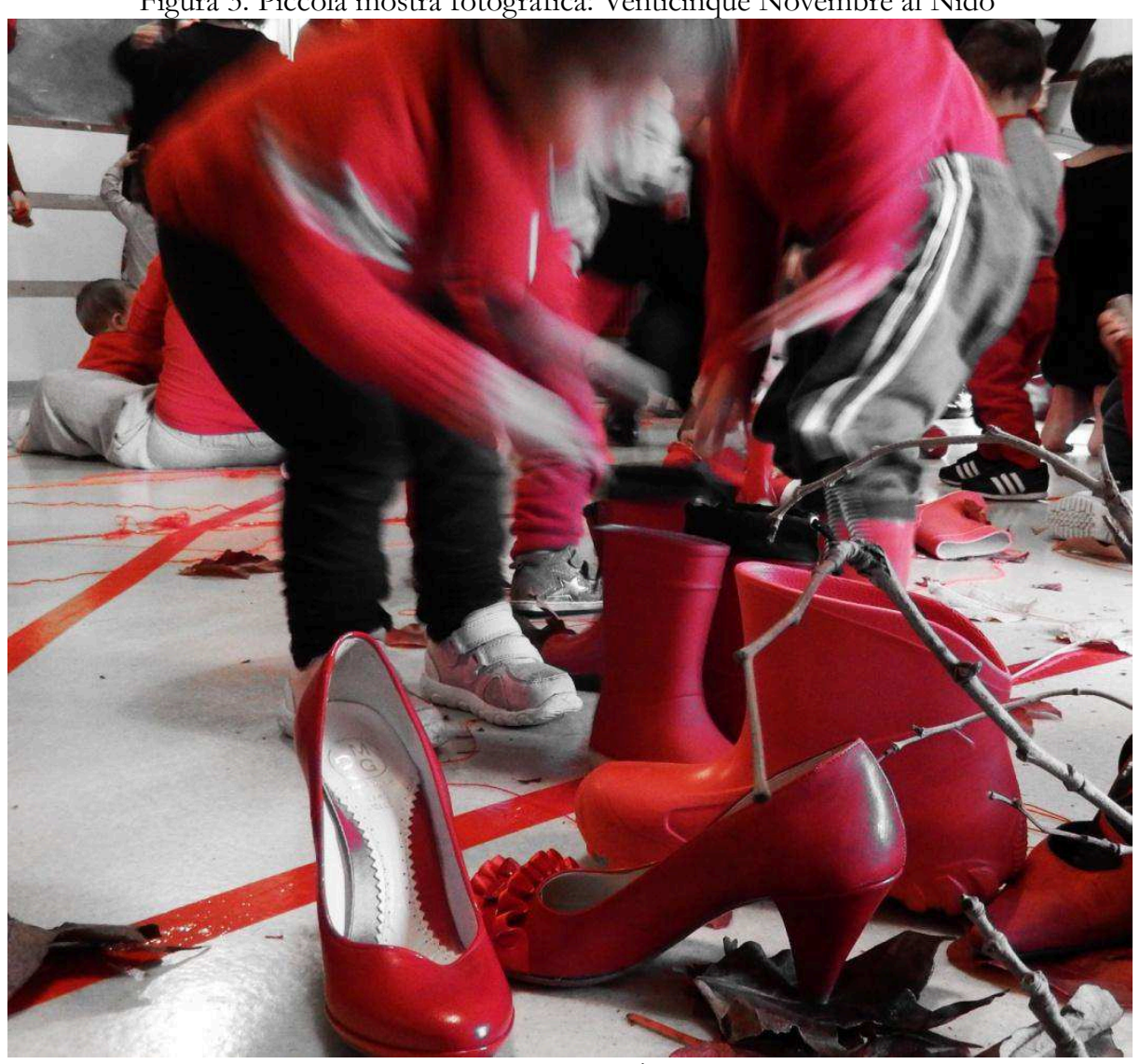

Fonte: Le autrice

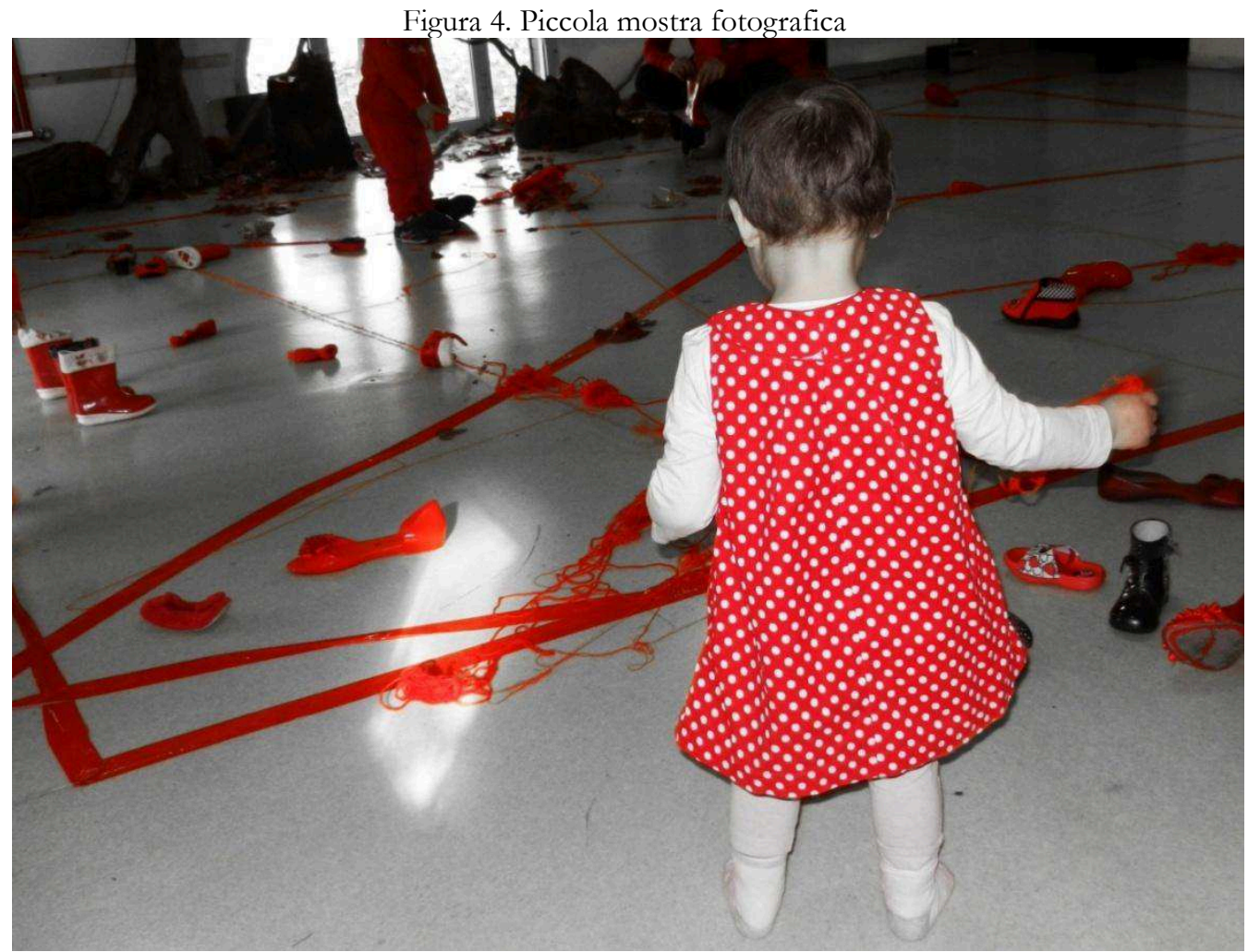

Fonte: Le autrice 
NIDO D'INFANZIA SAN DONATO

\section{ADESIONE ALLA GIORNATA INTERNAZIONALE CONTRO LA VIOLENZA SULLE DONNE}

\section{NOVEMBRE 2012}

"A noi non piace il gioco del silenzio. Non lo facciamo", evento all'interno del festival la violenza illustrata VII edizione "Economia domestica"

Il simbolo dell'iniziativa, a cui hanno partecipato tutti i nidi del quartiere San Donato, sono state le scarpe rosse e le impronte come segno delle donne e dei/le loro bambini/e che non ci sono più perché vittime della violenza di genere. Un messaggio che è voluto partire dai nidi per dire a tutte le donne di non sentirsi sole di fronte ad atti di violenza perpetrati nei loro confronti. I genitori hanno partecipato portando e posizionando su un filo un paio di scarpe rosse.

\section{NOVEMBRE 2013}

Un'iniziativa di sensibilizzazione sulla violenza di genere che parte dai dati sul femminicidio in Italia dove ogni due giorni viene uccisa una donna, altre subiscono violenza fisica o psicologica, i/le bambini/e sono vittime dirette o indirette di tali violenze. Simbolo delliniziativa è il colore rosso. Ai genitori abbiamo chiesto di appendere fuori dalle finestre o dai balconi delle loro abitazioni delle stoffe rosse, di vestirsi con magliette rosse, di collaborare al recupero di materiale rosso legato all'ambiente domestico (piatti, contenitori di plastica, strumenti da cucina, strofinacci, asciugamani...) inoltre di portare scarpe rosse o colorate di rosso. Genitori bambini/e e personale del nido hanno condiviso l'ascolto di musiche sul tema, camminato insieme sulle tracce lasciate dalle scarpe rosse da loro portate e hanno scoperto ed esplorato l'installazione...una grande scarpa creata con i materiali rossi. Al termine hanno condiviso il pranzo.

\section{NOVEMBRE 2014}

Non perdere il filo...rosso. Intrecci di storie da percorrere insieme. Evento all'interno del festival la violenza illustrata IX edizione "Happy hand"

Simbolo dell'iniziativa è il colore rosso. I genitori hanno collaborato al recupero di materiale rosso (gomitoli di lana, stoffe, maglioni, felpe, sciarpe, abiti) per la creazione di un'installazione/scenografia della performance presentata da educatrici del nido Valentina Aguzzi, Alba Indelicato, Elisabetta Martinelli, Lara Rocchetta una composizione di una trama narrativa dove il filo rosso è protagonista, creatrice di architetture corporee, ragnatele, dove il corpo dilata...ridisegna lo spazio. Intreccio di gesti, sguardi, movimenti. Un filo rosso che avvicina i/le bambini/e alla scoperta di materiali in un gioco di specchi che riflettono le tracce dei gesti e delle emozioni condivise. Al termine pranzo in comune. 


\section{NOVEMBRE 2015}

\section{Passi silenziosi raccontano tra i rami. Storie per ampliare lo sguardo e non perdere il filo...rosso.}

Evento all'interno del festival la violenza illustrata $\mathrm{X}$ edizione "Rinate di donna." Simbolo dell'iniziativa è il colore rosso. Scarpe di varie misure e gomitoli di lana rossi donati dai genitori sono materiale di scena, esplorazione e rielaborazione per la performance/laboratorio, presentata dalle educatrici Anna Catino, Elisabetta Martinelli, Valentina Aguzzi; Composizione di una trama narrativa dove il filo e le scarpe rosse raccontano la storia. Un filo di parole, un intreccio di passi tra fiaba e realtà. Il testo liberamente tratto da " C'era una volta una bambina " di Giovanna Zoboli e Joanna Concejo Ed. TopiPittori. Una partitura di passi per rendere visibile quello che è invisibile agli occhi Piccole spirali segnano lo spazio...l'energia del movimento risuona dentro di noi Gesti,movimenti,parole e suoni evocano immagini nascoste fra gli intrecci del corpo. Il suono del carillon e la circolarità della narrazione amplificano il nostro sentire e ci conducono nel bosco dove "lasciarsi spaventare" per aprire lo sguardo. Un filo rosso che avvicina i/le bambini/e alla scoperta di materiali ..scarpe,rami, possibili tracce,.. gesti che risuonano nei passi, movimenti che disegnano sentieri da percorrere insieme. L'eco delle storie risuona nel bosco riflettendo negli occhi e nei volti le immagini e le emozioni condivise. Conclusione con pranzo.

\section{NOVEMBRE 2016}

Sulla panchina impronte di parole. Incontri e sguardi raccontano. Iniziativa all'interno del festival la violenza illustrata XI edizione "Impasse partout"

Evento che unisce il colore rosso ad un altro elemento simbolo la panchina rossa. il segno tangibile di un assenza...del vuoto lasciato dalle donne vittime di femminicidio. "La panchina vuota è un porto d'affaccio sul mondo",I genitori hanno collaborato al recupero/creazione di panchine rosse per la presentazione della performance/laboratorio con le educatrici Elisabetta Martinelli e Valentina Aguzzi. Una trama narrativa dove la panchina, luogo di pausa silenzioso, rilascia gesti, movimenti, parole che svelano ricordi, memorie, segreti. Una partitura di attese e sguardi per rendere visibile quello che è invisibile agli occhi. Lo scorrere frenetico del tempo, scandito dal ritmo ripetuto di un metronomo, sulla panchina riprende respiro...rallenta, lo sguardo corre tra realtà e immaginazione, il movimento restituisce le storie... .parole sussurrate...brusii della notte...dell'ascolto Le panchine vuote...rifugi...Narrazioni dove il corpo...intreccia un filo rosso che si dipana tra le ombre del giardino. Il tempo si ferma...lo sguardo si apre...nell'immobilità..il sogno Le scarpe rosse che attraversano lo spazio lasciano segni da ripercorrere insieme bambini/e e adulti/e la panchina si offre per nuovi gesti,sguardi curiosi l'attraversano riflettono nel corpo movimenti ed emozioni,Le immagini scorrono lente raccontando storie nascoste nel paesaggio dell'infanzia. Conclusione con condivisione del pranzo.

Nella giornata del 24 una panchina donata dalla presidente del comitato di nido per essere collocata all'esterno e creare un ponte tra dentro e fuori, tra nido e territorio e ricordare che il femminicidio non è 
un fatto privato, ma collettivo, pubblico, è stata elemento di laboratorio espressivo per bambini/e che hanno lasciato tracce di colore rosso sulla panchina.

\section{NOVEMBRE 2017}

\section{Scarpette rosse...piccoli passi tra ragnatele di strade. Camminare per ampliare lo sguardo e}

ridisegnare lo spazio. Iniziativa all'interno del festival la violenza illustrata XII edizione "Ri-uscire"

Simbolo dell'iniziativa il colore rosso. Nello spazio esterno al nido viene realizzata un'istallazione con scarpe rosse, un richiamo al progetto Zapatos rojas di Elina Chauvet. Scarpe portate dai genitori e/o memoria di anni passati (quelle della fascia di età $0 / 12$ verranno donate alla Casa delle Donne per non subire violenza). In mattinata siamo usciti/e dal nido bambini/e e adulti/e e abbiamo iniziato a camminare lentamente, a piccoli passi, indossando scarpe rosse. Abbiamo percorso alcune strade del quartiere S. Donato, le stesse che alcune educatrici nelle giornate precedenti al calare della sera avevano percorso insieme e/o individualmente per sondare la percezione del pericolo nello spazio urbano dal loro punto di vista. Hanno camminato e mappato i luoghi con il corpo, condividendo pensieri, emozioni, sensibilità interrogandosi su quale ruolo gioca il corpo e la variabile di genere nell'attraversare i luoghi. Sulla base dell'esperienza è stato proposto un percorso attraverso strade, parchi e luoghi adiacenti il nido per riappropriarsi degli spazi, del piacere di camminare lentamente, rendere visibili i passi e sensibilizzare chi quotidianamente li attraversa. I nostri passi a volte hanno fermato il traffico restituendo il ritmo dell'infanzia alle strade, hanno ridisegnato spazi urbani con lenti respiri e battiti veloci. Emozioni condivise tra adulti/e e bambine/i. Corpi portatori di cultura e diversità. Ci siamo fermate in un giardino ascoltando la voce sospesa tra parole e poesie; dietro la sede del Quartiere San Donato abbiamo srotolato un lungo tappeto di giornali che molte volte nominano la violenza con un linguaggio che non la riconosce e con immagini e parole stereotipate che non leggono i cambiamenti culturali e lo status delle donne, abbiamo segnato con nuovi passi il tappeto calpestando simbolicamente l'informazione stereotipata e tendenziosa che spesso la stampa propone nell'affrontare la violenza di genere. Il camminare crea un flusso continuo di attraversamenti, lo scorrere del tempo sul tapis roulant ritma la voce e narra l'arte del passato.

Nell'area davanti al quartiere i cellulari, segno del presente che avanza, sincronizzati echeggiano le note del brano Think, e la voce di Aretha Franklin si diffonde, nel cerchio, la danza dei corpi libera l'energia. Continuiamo a camminare per raggiungere lo spazio antistante l'associazione culturale GRAF S. Donato. Sedute/i su un blocco di cemento, lo sguardo si posa su corpi di donne in movimento, che resistenti gridano il loro nome, gesti ripetuti segnano lo spazio, riaffermando identità che la violenza vuole annullare. Una performance dove la violenza agita sulle donne diventa rafforzamento, autodeterminazione, libertà. Cinque donne percorrono lo spazio, improvvisamente si fermano e i loro corpi si abbassano fino a terra schiacciati lentamente; si rialzano, poi nuovamente a terra e poi sempre con maggior forza e velocità, si alzano affermando il loro nome, la loro "esistenza". Adulte/i e bambine/i guardano in silenzio, 
osservano... alcuni bambini/e entrano nello spazio, creano con il corpo, emozioni fissate nella memoria attraverso sguardi condivisi.

A piccoli passi continuiamo a camminare, raggiungiamo lo spazio davanti al Nido dove scarpe rosse simbolo di presenza-assenza-traccia da percorrere ci attendono. Fili invisibili, ragnatele di strade, intrecci di sguardi segnano nuove tracce, fili sospesi tra realtà e immaginazione. Una trama sottile, un'intricata tela che rappresenta il tema della violenza maschile sulle donne, una trama sistematica frequente in cui si può restare invischiate, la difficoltà nel vedere i fili e uscire dalla ragnatela,ma anche la capacità delle donne di creare connessioni tra di loro e fili capaci di incrociare le diverse soggettività. Per noi al nido ha inoltre rappresentato la connessione tra le donne e gli uomini presenti in quella giornata, lintreccio quotidiano tra adulti/e e bambini/e, il patto educativo, il legame forte anche se intangibile di chi si impegna per ri-uscire. Abbiamo pensato di ridisegnare lo spazio dedicando i luoghi di sosta agiti a donne che hanno subito violenza di genere e/o che con la loro autodeterminazione e professionalità hanno contribuito a rendere visibile la violenza subita da altre e che quotidianamente e attivamente re-sistono. La scelta e la riflessione sui nomi è nata a partire dalla toponomastica presente nel nostro quartiere, nomi legati al mondo dell'arte, del teatro, donne/uomini e all'intreccio delle nostre quotidianità...

Attenzione costante ai diversi linguaggi espressivi. Ogni luogo un nome, un'artista, una donna: Susanna Chavez (Ciudad Juàrez 5/11/1974-Ciudad Juàrez 6/01/2011), Artemisia Gentileschi(Roma 8/07/1593Napoli dopo 31/01/1654, Franca Rame (Parabiago 18/07/1929-Milano 29/05/2013), Zanele Muholi (Umlaz-DurbanSudafrica 19/07/1972), Luki Massa (1962-Bologna 06/09/2016).

Ogni volta che una voce di donna si leva per leggere la biografia di una donna, il silenzio si amplifica nell'ascolto, le emozioni segnano i volti. Siamo rientrati/e al nido per condividere il momento del pasto, rigorosamente rosso... pasta al pomodoro. Stemperate le emozioni condivise tra bambini/e e adulti/e abbiamo concluso la mattinata recandoci nello spazio antistante l'ingresso del nido per inaugurare la panchina rossa contro il feminicidio; un ponte tra interno ed esterno, un luogo dove fermarsi, riflettere e sensibilizzare sul tema della violenza sulle donne. La panchina è stata realizzata dall'artista Dizz Jesus Mercedes, insieme alle/i bambine/i,un momento laboratoriale condiviso con le educatrici, i gesti e le tracce dell'esperienza hanno segnato e dipinto di colore rosso l'elemento simbolico. Questo spazio è stato dedicato a una bambina vittima di femminicidio: Sara Jay Cusmà Piccione (1992-Bologna 19/04/2001)

Negli anni il confronto con i genitori nell'affrontare il tema della violenza di genere all'interno del nido ha evidenziato nelle loro parole la complessità e la difficoltà di trattare quello che racchiude, con delicatezza e in modo adeguato.

Le restituzioni dei genitori sulla giornata hanno rafforzato in noi la convinzione della necessità di esprimere tale complessità attraverso diversi linguaggi/codici, espressivi/artistici; linguaggi capaci di aprire sguardi differenti, suscitare pensieri profondi, emozioni che sedimentano nella memoria di bambini/e e adulti/e. Racchiudere significati, messaggi, intrecciare realtà e immaginazione per accostare bambini/e e adulti/e ad un tema complesso per educare alla non violenza e al rispetto dell'altro/a. Ridisegnare percorsi 
avviando nuove connessioni basate su un'educazione in contrasto alla violenza di genere, un'attenzione costante alle relazioni, agli stereotipi di genere ad un linguaggio capace di riconoscere le differenze, e non si esprima nel maschile neutro e universale. Un percorso in divenire, un'iniziativa che dal nido si apre alla cittadinanza, al territorio per sradicare la violenza sistemica contro le donne. Un'iniziativa per promuovere una cultura dell'infanzia che vive e si alimenta in sguardi condivisi, un'energia che non si nasconde, ma si disvela, intrecciando fili che creano trame future. Un progetto educativo che parte dal quotidiano, dallo $0 / 3$, per prevenire la violenza di genere.

"Abbiamo bisogno della "tempesta perfetta" intorno al tema della violenza di genere: un cambiamento di atteggiamenti culturali, la fine del silenzio e della connivenza che spesso accompagnano il senso di diritto acquisito di un uomo di controllare la sua compagna e una radicale rieducazione delle nostre comunità. Alla base di tutti questi cambiamenti necessari è il bisogno di affrontare le molte aree della disuguaglianza che ancora esistono per le donne di oggi" (Catherine Dunne , "Nuove grammatiche dell'amore") 